\title{
Urinary Exosomes and Exosomal CCL2 mRNA as Biomarkers of Active Histologic Injury in IgA Nephropathy
}

Ye Feng, ${ }^{*}$ Lin-Li Lv, ${ }^{*}$ Wei-Jun Wu, ${ }^{*}$ Zuo-Lin Li, ${ }^{*}$ Jun Chen, * Hai-Feng Ni, * Le-Ting Zhou, * Tao-Tao Tang, * Feng-Mei Wang, * Bin Wang, ${ }^{*}$ Ping-Sheng Chen, ${ }^{*}$ Steven D. Crowley, ${ }^{\dagger}$ and Bi-Cheng Liu*

From the Institute of Nephrology, ${ }^{*}$ Zhongda Hospital, Southeast University School of Medicine, Nanjing, China; and the Division of Nephrology, ${ }^{\dagger}$ Department of Medicine, Duke University, Durham VA Medical Centers, Durham, North Carolina

Accepted for publication

July 24, 2018.

Address correspondence to Bi-Cheng Liu, M.D., Ph.D., or Lin-Li Lv, M.D., Ph.D., Institute of Nephrology, Zhongda Hospital, Southeast University School of Medicine, 87 Ding Jia Qiao Rd., Nanjing, China. E-mail: liubc64@163.com or1vlinli@seu.edu.cn.

\begin{abstract}
IgA nephropathy (IgAN) features variable renal pathology and a heterogeneous clinical course. Our aim was to search noninvasive biomarkers from urinary exosomes for IgAN patients; membrane nephropathy and minimal change disease were included as other glomerulopathy controls. Transmission electron microscopy and nanoparticle tracking analysis confirmed the size and morphology characteristic of urinary exosomes. Exosome markers (Alix and CD63) as well as renal cell markers [aquaporin 2 (AQP2) and nephrin] were detected, which indicate the renal origin of urinary exosomes. Exosome excretion was increased markedly in IgAN patients compared with controls and correlated with levels of proteinuria and tubular injury. More important, urinary exosome excretion correlated with greater histologic activity (mesangial hypercellularity, crescents, and endocapillary hypercellularity). Profiling of the inflammation-related mRNA revealed that exosomal chemokine (C-C motif) ligand 2 (CCL2) was upregulated in IgAN patients. In a validation study, CCL2 was exclusively highly expressed in IgAN patients compared with healthy controls as well as minimal change disease and membrane nephropathy patients. Also, a correlation between exosomal CCL2 and estimated glomerular filtration rate levels was found in IgAN. Exosomal CCL2 was correlated with tubulointerstitial inflammation and C3 deposition. High CCL2 levels at the time of renal biopsy were associated with subsequent deterioration in renal function. Thus, urinary exosomes and exosomal CCL2 mRNA are promising biomarkers reflecting active renal histologic injury and renal function deterioration in IgAN. (Am J Pathol 2018, 188: 2542-2552; https://doi.org/10.1016/j.ajpath.2018.07.017)
\end{abstract}

$\operatorname{Ig}$ A nephropathy ( $\operatorname{Ig} \mathrm{AN})$ is one of the most common primary renal diseases worldwide and is an important cause of end-stage renal disease. ${ }^{1}$ However, patients with IgAN show variable clinical presentations, which range from asymptomatic microscopic hematuria to rapidly progressive glomerulonephritis or nephrotic syndrome.,3 Besides, although $\operatorname{IgAN}$ runs a relatively benign course in most patients, up to $40 \%$ of patients progress to end-stage renal disease over the course of 30 to 40 years. Thus, biomarkers for predicting outcomes and identifying patients at higher risk of progression are essential for better management of IgAN.

Currently, renal biopsy and pathologic assessment remain the standard diagnostic approach in $\operatorname{IgAN}{ }^{1,4}$ Biopsy specimens meeting IgAN diagnostic criteria have a range of pathologic changes that are reflected in the variable clinical course of IgAN. $^{3}$ Given the invasive nature and nontrivial postoperative complications of biopsy, novel noninvasive biomarkers associated with kidney histologic damage are urgently needed for clinical practice.

Supported by National Natural Scientific Foundation grants 81470922 (L.-L.L.), 31671194 (L.-L.L.), 81720108007 (B.-C.L.), and 81670696 (B.-C.L.), Clinic Research Center of Jiangsu Province grant BL2014080 (B.-C.L.), and Jiangsu Province Medical Youth Talent grant QNRC2016818 (L.-L.L.).

Y.F. and L.-L.L. contributed equally to this work.

Disclosures: None declared. 
Exosomes are nano-sized extracellular vesicles of endosomal origin, secreted into the extracellular space through fusion of multivesicular bodies with the plasma membrane. ${ }^{5,6}$ Urine has been a promising source for identifying novel biomarkers for kidney disease. ${ }^{7}$ Since the first description of exosomes in the urine in $2004,{ }^{8}$ urinary exosomes have become an attractive target for biomarker discovery in kidney disease. Urinary exosomes may be produced by epithelial cells facing the urinary space, including podocytes and renal tubular epithelial cells. ${ }^{8,9}$ Thus, the urinary exosome could represent a promising liquid biopsy that reflects the pattern and/or severity of renal histologic injury. The discovery of exosomes as natural carriers of RNA (miRNA and mRNA) information from parent cells has raised great interest in exosomal RNAs as potential biomarkers for renal disease. CD2AP mRNA and miR-29c in urinary exosomes may be used as biomarkers that reflect chronic kidney disease progression and the severity of fibrosis. ${ }^{10,11}$ Other laboratories have shown that exosomal miRNA may also be a potential biomarker of chronic kidney disease, diabetic kidney disease, and lupus nephritis..$^{9,12-14}$ However, the role of urinary exosomal RNAs in the monitoring of IgAN has not been tested.

Tubular and interstitial inflammation plays a crucial role not only in the onset but also in the progression of IgAN. ${ }^{15}$ Numerous lines of evidence have demonstrated that an immunohistochemical evaluation of tubulointerstitial inflammation is a powerful tool in determining the prognosis in IgAN. ${ }^{16-18}$ Inflammatory responses are involved in the pathogenesis of $\operatorname{IgAN}$ at distinct phases and in characteristic lesions, possibly via the recruitment and activation of distinct types of leukocyte populations. ${ }^{19}$ Interestingly, the exosomal chemokine (C-C motif) ligand 2 (CCL2) plays an important role in the development of tubulointerstitial inflammation. ${ }^{20}$ In this study, we profiled the expression of a panel of cytokines and chemokines as well as their receptors in urinary exosomes to evaluate their potential role as noninvasive biomarkers of IgAN. Patients with IgAN showed augmented urinary excretion of exosomes with exaggerated expression of CCL2 mRNA compared with other molecules. Further study showed that $C C L 2$ mRNA levels correlated with more severe histologic activity and tubular injury. More important, the exosomal CCL2 mRNA may represent a promising biomarker for deterioration of renal function and active tubulointerstitial injury in IgAN.

\section{Materials and Methods}

\section{Patients and Normal Controls}

For detection of exosomal inflammation-related mRNA expression, in the preliminary screening test, a group of six IgAN patients and six healthy controls were enrolled. In the validation study, 55 patients with IgAN and 24 normal
Table 1 Human Subject Characteristics of Healthy Controls and IgAN Patients

\begin{tabular}{lll}
\hline Characteristic & $\begin{array}{l}\text { Healthy controls } \\
(n=24)\end{array}$ & $\begin{array}{l}\text { IgAN patients } \\
(n=55)\end{array}$ \\
\hline Male/female ratio & $11: 13$ & $31: 24$ \\
Age, years & $38.2 \pm 13.8$ & $38.6 \pm 13.3$ \\
$\mathrm{BUN}, \mathrm{mmol} / \mathrm{L}$ & $4.15(3.53-4.96)$ & $5.70(4.40-7.96)^{* * *}$ \\
$\mathrm{SCr}, \mu \mathrm{mol} / \mathrm{L}$ & $78(68.25-89.5)$ & $97(73.5-127.5)^{*}$ \\
$\mathrm{UA}, \mu \mathrm{mol} / \mathrm{L}$ & $307(274-341)$ & $349(276-418)$ \\
$\mathrm{eGFR}, \mathrm{mL} / \mathrm{minute}^{*}$ & $86.2(78.7-97.3)$ & $73.8(46.7-91.9)^{*}$ \\
$\quad \begin{array}{l}\text { per } 1.73 \mathrm{~m}^{2} \\
\text { Proteinuria, }\end{array}$ & & \\
$\mathrm{g} / 24$ hours & $<0.3$ & $2.45(1.32-5.45)^{*}$ \\
\hline
\end{tabular}

eGFR has been calculated using the modification of diet in renal disease creatinine formula $\left(\mathrm{mL} /\right.$ minute per $\left.1.73 \mathrm{~m}^{2}\right)$. Data are expressed as means \pm SD or median (interquartile range).

${ }^{*} P<0.05,{ }^{* * *} P<0.001$ (U-test).

BUN, blood urea nitrogen; eGFR, estimated glomerular filtration rate; IgAN, IgA nephropathy; SCr, serum creatinine; UA, uric acid.

individuals were included. In addition to healthy controls, patients with membranous nephropathy (MN; $n=16)$ or minimal change disease (MCD; $n=9)$ were recruited as other glomerulopathy controls. All cases of IgAN were proved by biopsy in the Kidney Institute of the Zhongda Hospital, Southeast University (Nanjing, China). The inclusion criteria included the following: patients with biopsyproved IgAN and age $\geq 18$ years. The exclusion criteria were as follows: patients with malignancy or severe liver injury; patients who were pregnant or in the lactation period; patients who had undergone renal replacement therapy, such as kidney transplantation or dialysis; and patients with acute or chronic infection. The glomerular filtration rate was estimated by the modification of diet in renal disease equation. Twenty-four normal individuals were included without proteinuria, hypertension, diabetes, impaired renal function, or chronic inflammatory diseases. The baseline clinical characteristics, including blood urea nitrogen, serum creatinine, uric acid, proteinuria, and urinary neutrophil gelatinase-associated lipocalin, were recorded. All of the studies were approved by the Ethics Committee of Zhongda Hospital, Southeast University.

Urine and blood samples were collected from $36 \operatorname{IgAN}$ patients after renal biopsy, with a median of 18.5 months of follow-up.

\section{Urine Sample Preparation and Exosome Isolation}

The first morning urine was collected. Samples were centrifuged for 20 minutes at $2000 \times g$ and $13,500 \times g$ at $4^{\circ} \mathrm{C}$ (model 5804R; Eppendorff, Hamburg, Germany) to remove cellular debris and eliminate large microvesicles, respectively. Supernatants were then ultracentrifuged for 2 hours at $200,000 \times g$ at $4^{\circ} \mathrm{C}$ (Beckman Instruments, Fullerton, CA), and urinary exosomes were resuspended in 100 
$\mu \mathrm{L}$ phosphate-buffered saline. The pellet was frozen at $-80^{\circ} \mathrm{C}$ for later analysis.

\section{Exosome Characterization and mRNA Measurement}

The exosome pellets were diluted five times with phosphatebuffered saline and then applied to 200-mesh nickel grids. Samples were stained with $2 \%$ phosphotungstic acid for 5 minutes and air dried. Exosomes were detected using a transmission electron microscope (HT 7700; Hitachi HighTech, Tokyo, Japan) at $80 \mathrm{kV}$. ZetaView Nanoparticle Tracking analysis (Particle Metrix, Meerbusch, Germany) was performed, as reported before. ${ }^{20}$ The protein from exosomes was extracted by RIPA Lysis and Extraction Buffer (Thermo, Wilmington, DE) with protease inhibitor and phosphatase inhibitor. Urine exosome was quantified using the EXOCET assay (System Biosciences, Mountain View, CA), which measured the activity of exosome acetylcholinesterase. $^{21}$ Exosome quantification was also normalized to urine creatinine to control for variation in urine flow rate.
Exosome markers (Alix and CD63) were detected by Western blot analysis. Antibodies, including mouse anti-Alix (1:500; Santa Cruz Biotechnology, Dallas, TX), mouse anti-CD63 (1:1000; Abcam, Cambridge, MA), goat antinephrin (1:400; Santa Cruz Biotechnology), and mouse anti-AQP2 (1:500; Santa Cruz Biotechnology), were used.

Total RNA was extracted from urinary exosomes with the miRNeasy micro kit (Qiagen, Hilden, Germany), according to the manufacturer's protocol. PrimeScript RT Reagent Kit with gDNA Eraser (Takara, Kusatsu, Japan) was used for reverse transcription, according to the manufacturer's protocol. Urinary exosomal mRNA levels were quantified by real-time RT-PCR on the ABI Prism 7300 Sequence Detection System (Applied Biosystems, Foster City, CA).

\section{Evaluation of Renal Histopathological Lesions}

Two experienced pathologists (P.-S.C. and H.-F.N.) who were masked to the patients' information performed the assessment of the histopathological damage using the
A

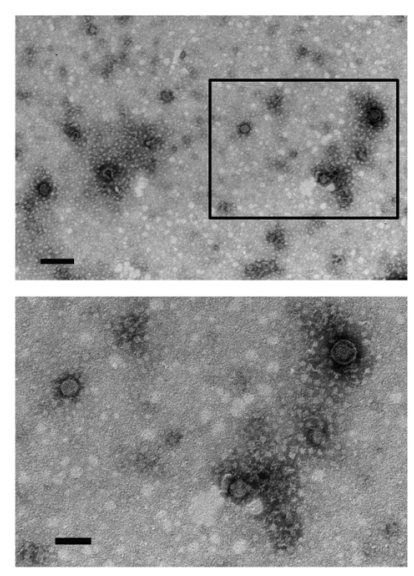

D

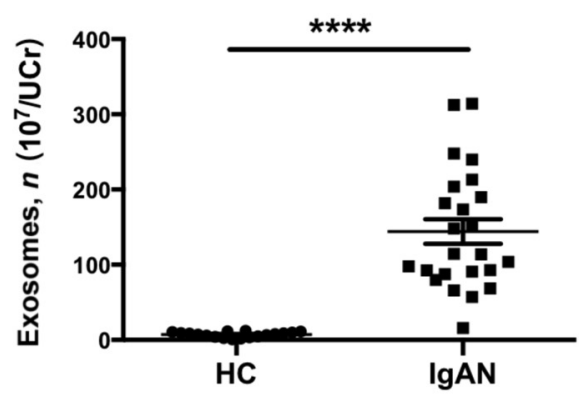

B

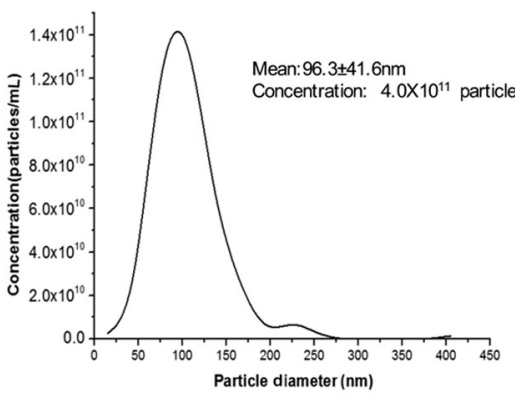

E
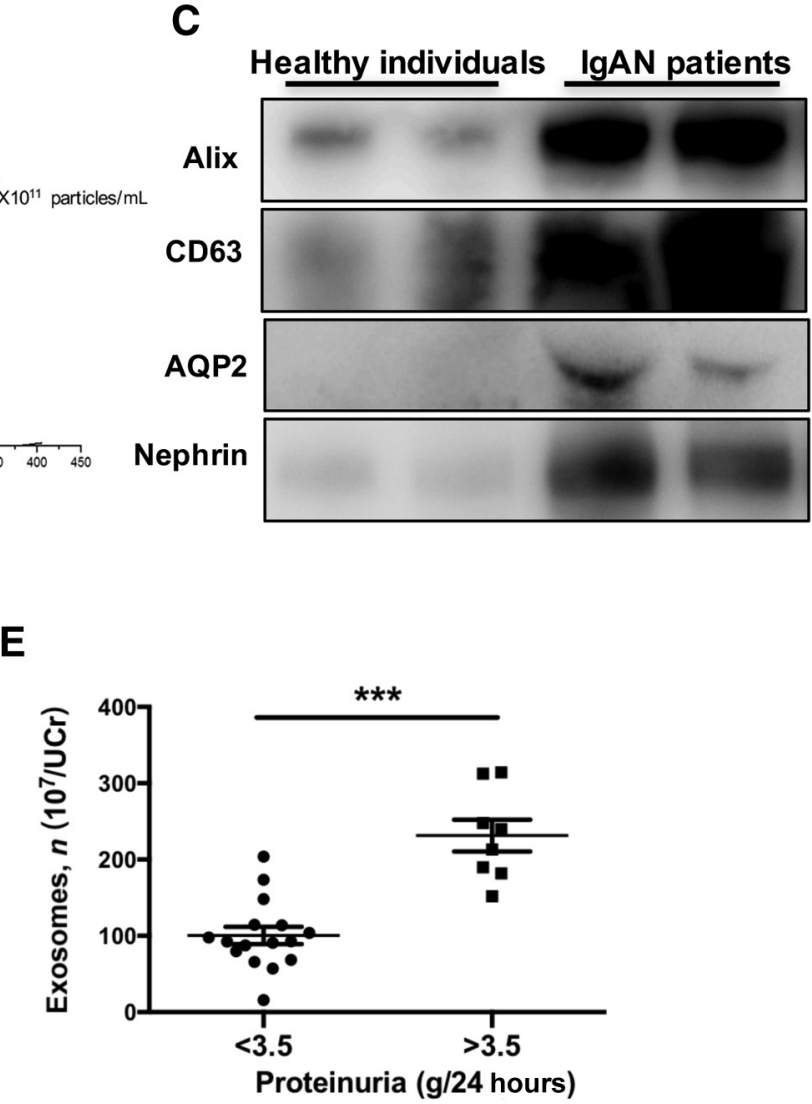

Figure 1 Urinary exosome excretion is increased in IgA nephropathy (IgAN) patients. A: Representative electron micrograph of exosomes in urine from IgAN patients. Boxed area is shown at higher magnification below. B: Size distribution of urinary exosomes from IgAN patients analyzed by nanoparticle tracking analysis. The mean diameter of exosomes was $96.3 \pm 41.6 \mathrm{~nm}$. C: Expression of exosomal markers (Alix and CD63) and renal cell markers (AQP2 and nephrin) was assessed by Western blot analysis in protein extracted from isolated urinary exosomes of healthy controls and IgAN patients. Note a significant increase in urinary exosome excretion normalized to urine creatinine ( $\mathrm{UCr}$ ) in IgAN patients. D: Urinary exosomal protein was quantified by EXOCET assay, which measured the activity of exosome acetylcholinesterase, and results were normalized to UCr in 15 healthy controls (HCs) and 29 biopsy-proven IgAN patients. Urinary exosome protein production was significantly increased in IgAN patients. E: The amount of exosomes was markedly increased in the cohort of severe proteinuria ( $>3.5 \mathrm{~g} / 24$ hours) compared with patients with nonsevere proteinuria ( $\leq 3.5 \mathrm{~g} / 24$ hours). ${ }^{* * *} P<0.001, * * * \star P<0.0001$. Scale bars: 200 $\mathrm{nm}$ (A, top panel); $100 \mathrm{~nm}$ (A, bottom panel). 

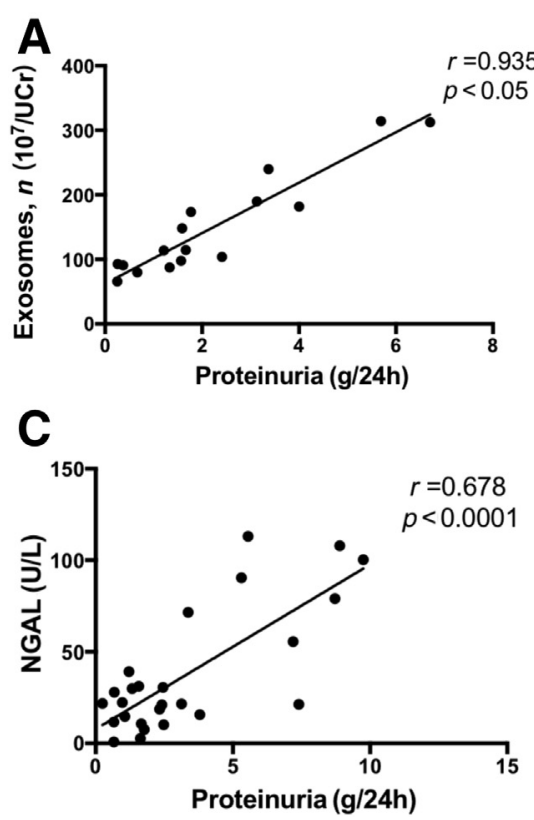

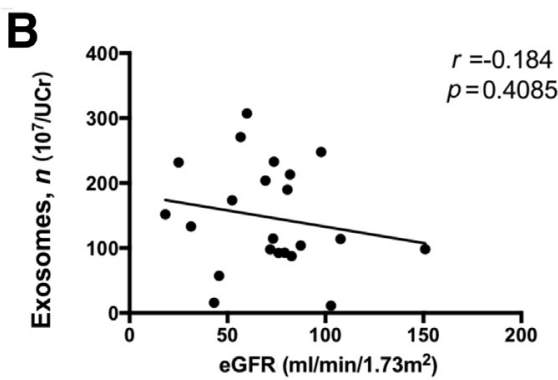

D

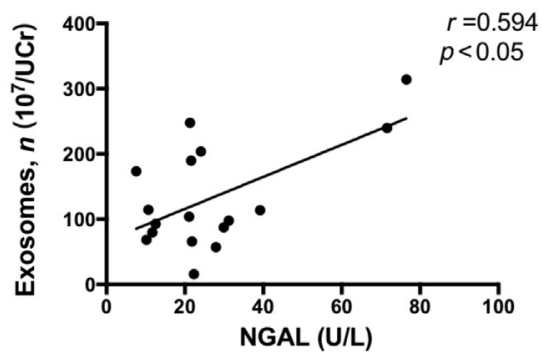

Figure 2 Urinary exosome excretion is correlated with levels of proteinuria and tubular injury in IgA nephropathy (IgAN) patients. A: Normalized concentration of urinary exosome amount in IgAN patients positively correlates with levels of proteinuria at the time of renal biopsy. B: No significant correlation is found between urinary exosome excretion and estimated glomerular filtration rate (eGFR) in IgAN patients. C: Plots of correlation between neutrophil gelatinaseassociated lipocalin (NGAL) and proteinuria in IgAN patients. D: Normalized urinary exosome quantification correlates closely with levels of NGAL in IgAN patients. Lines show the linear correlation for each of the two variables. $\mathrm{UCr}$, urine creatinine. updated Oxford MEST-C scoring system. ${ }^{22}$ The mesangial score was recorded as the number of cells per mesangial area (M1 or M0). The endocapillary hypercellularity was scored as presence (E1) or absence (E0). The segmental sclerosis score was measured as absent (S0) or present (S1). Interstitial fibrosis or tubular atrophy was classified according to the area with tubulointerstitial damage: $<25 \%$ (T0), $25 \%$ to $50 \%$ (T1), or $>50 \%$ (T2). ${ }^{22}$ Tubulointerstitial inflammation was semiquantitatively graded into normal (no significant infiltrations), mild (up to three local infiltrations involving up to $25 \%$ of the interstitium), and marked (any local infiltrations involving $>25 \%$ of interstitium or diffuse infiltration). ${ }^{16,18} \mathrm{C} 3$ deposition was observed and scored as follows:,,-+++ , and +++ . Crescent lesions were scored as $\mathrm{C} 0$ (no crescents), $\mathrm{C} 1$ (crescents in $<25 \%$ of glomeruli), and $\mathrm{C} 2$ (crescents in $\geq 25 \%$ of glomeruli).

Infiltration of macrophages within the kidney was demonstrated by immunoperoxidase staining of CD68. The primary monoclonal antibodies used were anti-CD68 antibodies (1:100; ab-201340; Abcam).
A
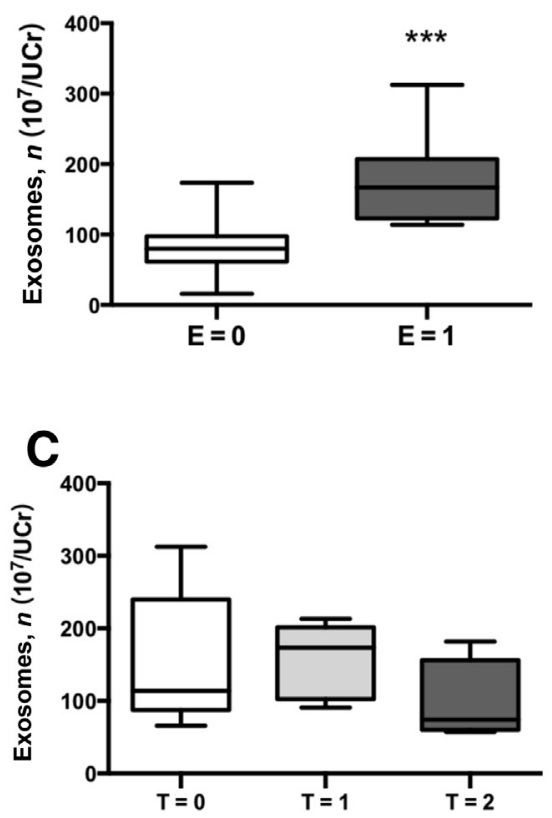

B

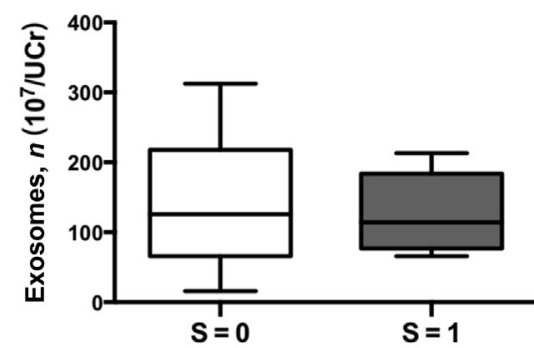

D

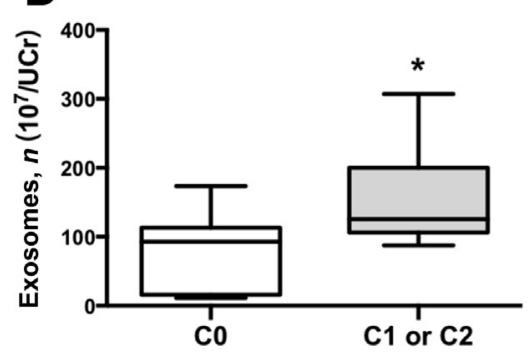

Figure 3 Correlation between urinary exosome excretion and kidney histologic injury. A: Patients with endocapillary hypercellularity $(E=1)$ show increased urinary exosome production compared with those without endocapillary hypercellularity $(E=0)$. B: No differences in normalized urinary exosome are found between IgA nephropathy patients with $(S=1)$ or without $(S=0)$ segmental glomerulosclerosis. C: No difference is found in patients with different tubular atrophy and interstitial fibrosis severity [area with tubulointerstitial damage: $<25 \%(T=0), 25 \%$ to $50 \%(T=1)$, or $>50 \%(\mathrm{~T}=2)$ ]. D: Patients with crescents (C1/ C2) show increased exosome excretion compared with patients without crescents (C0). ${ }^{*} P<0.05$, $* * * P<0.001$ (t-test). $U C r$, urine creatinine. 
Table 2 Human Subject Characteristics of Healthy Controls and IgAN Patients for Screening of Inflammation-Related Genes in Urinary Exosomes

\begin{tabular}{lcl}
\hline Characteristic & Healthy controls & IgAN patients \\
\hline Male:female, $n$ & $3: 3$ & $3: 3$ \\
Age, years & $27(26.7-30.5)$ & $36.5(22.3-42.5)$ \\
$\mathrm{BUN}, \mathrm{mmol} / \mathrm{L}$ & $3.5(3.4-3.8)$ & $5.15(3.37-9.13)$ \\
$\mathrm{SCr}, \mu \mathrm{mol} / \mathrm{L}$ & $64.5(59.0-75.7)$ & $98.5(79.0-128.7)^{*}$ \\
$\mathrm{UA}, \mu \mathrm{mol} / \mathrm{L}$ & $255(209-287)$ & $314(266-374)$ \\
$\mathrm{eGFR}, \mathrm{mL} / \mathrm{minute}^{*}$ & $99.1(83.7-114.2)$ & $74.3(50.1-91.7)^{*}$ \\
$\quad \mathrm{per} 1.73 \mathrm{~m}^{2}$ & & \\
Proteinuria, & $<0.3$ & $1.41(0.78-7.57)^{*}$ \\
$\quad \mathrm{~g} / 24$ hours & & \\
\hline
\end{tabular}

Values are expressed as median (interquartile range).

${ }^{*} P<0.05$ (U-test).

BUN, blood urea nitrogen; eGFR, estimated glomerular filtration rate; IgAN, IgA nephropathy; SCr, serum creatinine; UA, uric acid.

\section{Statistical Analysis}

Statistical analysis was conducted by SPSS version 18.0 (SPSS Inc., Chicago, IL) and GraphPad Prism 5 (GraphPad Software, San Diego, CA). All of the data were shown as means \pm SD or median (interquartile range), whether the data were normally or abnormally distributed. The relative amount of mRNA to glyceraldehyde-3-phosphate dehydrogenase was described by the equation $\left(2^{-\Delta \mathrm{Ct}}\right)$, where $\Delta \mathrm{Ct}$ refers to Ct-mRNA minus $\mathrm{Ct}$-glyceraldehyde-3-phosphate dehydrogenase. In the screening analysis, the value of $2^{-\Delta \mathrm{Ct}}$ was $\log 2$ transformed and analyzed by the heat map package in $\mathrm{R}$ version 3.2.5. $t$-Test, $U$-test for non-parametric data, or one-way analysis of variance was used to compare the data between different groups. Spearman's correlation was performed to analyze the correlation between two groups of data.

\section{Results}

\section{Urinary Exosome Excretion Is Increased in IgAN Patients}

Exosomes were purified from the urine of 55 IgAN patients and 24 healthy controls matched by age and sex. The basic clinical characteristics of these patients are listed in Table 1. IgAN patients showed significantly higher levels of blood urea nitrogen, serum creatinine, and proteinuria, with reduced values of estimated glomerular filtration rate (eGFR), compared with healthy controls $(P<0.05)$.

Urinary exosomes were characterized by using transmission electron microscopy, ZetaView nanoparticle tracking analysis, and Western blot analysis (using Alix and CD63 as exosome markers). Transmission electron microscopy showed homogeneous size of vesicles, and the diameter was $96.3 \pm 41.6 \mathrm{~nm}$, as measured by nanoparticle tracking analysis (Figure 1, A and B). Exosome markers Alix and CD63 were readily detected by Western blot analysis. More important, tubular epithelial marker AQP2 and podocyte marker nephrin were detected in urinary exosomes, which confirmed the renal origin of the purified exosomes. Interestingly, urinary exosome excretion was significantly enhanced in IgAN patients compared with

Table 3 Primers for Quantitative RT-PCR

\begin{tabular}{|c|c|c|}
\hline Primer & Forward & Reverse \\
\hline Homo CCL-2 & $5^{\prime}$-ССТTCATTCCCCAAGGGCTC-3' & 5'-GGTTTGCTTGTCCAGGTGGT-3' \\
\hline Homo CCL-3 & $5^{\prime}-$ TCTTCCTAACCAAGCGAAGC-3' & 5'-TTGGCAACAACCAGTCCATA-3' \\
\hline Homo CCL-21 & $5^{\prime}-\mathrm{AGAGAGACCGAGGAGGGAGA}-3^{\prime}$ & $5^{\prime}-$ TTTGGGTGGGAAGACAGAAC- $3^{\prime}$ \\
\hline Homo CCL-22 & $5^{\prime}$-CTGTTCCCATCAGCGATTCC-3' & $5^{\prime}$-GACCCAGAAGTGGGATGTGT-3' \\
\hline Homo CXCL-1 & $5^{\prime}-$ ACTCAAGAATGGGCGGAAAG- $3^{\prime}$ & $5^{\prime}-$ TCCTAAGCGATGCTCAAACA-3' \\
\hline Homo CXCL-10 & $5^{\prime}-\mathrm{AAGGATGGACCACACAGAGG-3^{ \prime }}$ & $5^{\prime}-\mathrm{AGCAGGGTCAGAACATCCAC-3^{ \prime }}$ \\
\hline Homo CCR2 & 5'-CGGTGCTCCCTGTCATAAAT-3' & $5^{\prime}-\mathrm{CCCAAAGACCCACTCATTTG-3^{ \prime }}$ \\
\hline Homo CCR5 & $5^{\prime}$-AACCAGGCGAGAGACTTGTG-3' & $5^{\prime}$-CAATGCCGTCAAGGTTCTTC-3' \\
\hline Homo CXCR-2 & 5'-TCAGTGTGGACCGTTACCTG-3' & $5^{\prime}$-CACGATGAAGCCAAAGGACT-3' \\
\hline Homo CXCR-4 & $5^{\prime}-$ TCATCAAGCAAGGGTGTGAG-3' & $5^{\prime}$-GAGAGGATCTTGAGGCTGGA-3' \\
\hline Homo IL-22 & $5^{\prime}$-AGGCTCAGCAACAGGCTAAG-3' & 5'-TTCAGCTTTGCTCTGGTCAA-3' \\
\hline Homo IFN- $\gamma$ & 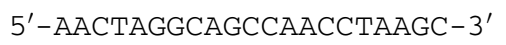 & $5^{\prime}-$ AAGCACCAGGCATGAAATCT-3' \\
\hline Homo TNF- $\alpha$ & $5^{\prime}$-CCCAGGGACCTCTCTCTAATC-3' & $5^{\prime}-\mathrm{TGAGGTACAGGCCCTCTGAT-3^{ \prime }}$ \\
\hline Homo GAPDH & 5'-CTCTGCTCCTCCTGTTCGAC-3' & $5^{\prime}$-GCGCCCAATACGACCAAATC-3' \\
\hline
\end{tabular}

$\mathrm{CCL}$, chemokine (C-C motif) ligand; $\mathrm{CXCR}, \mathrm{C}-\mathrm{X}-\mathrm{C}$ chemokine receptor; $\mathrm{GAPDH}$, glyceraldehyde-3-phosphate dehydrogenase; Homo, homo sapiens; IFN- $\gamma$, interferon- $\gamma$; TNF- $\alpha$, tumor necrosis factor- $\alpha$. 


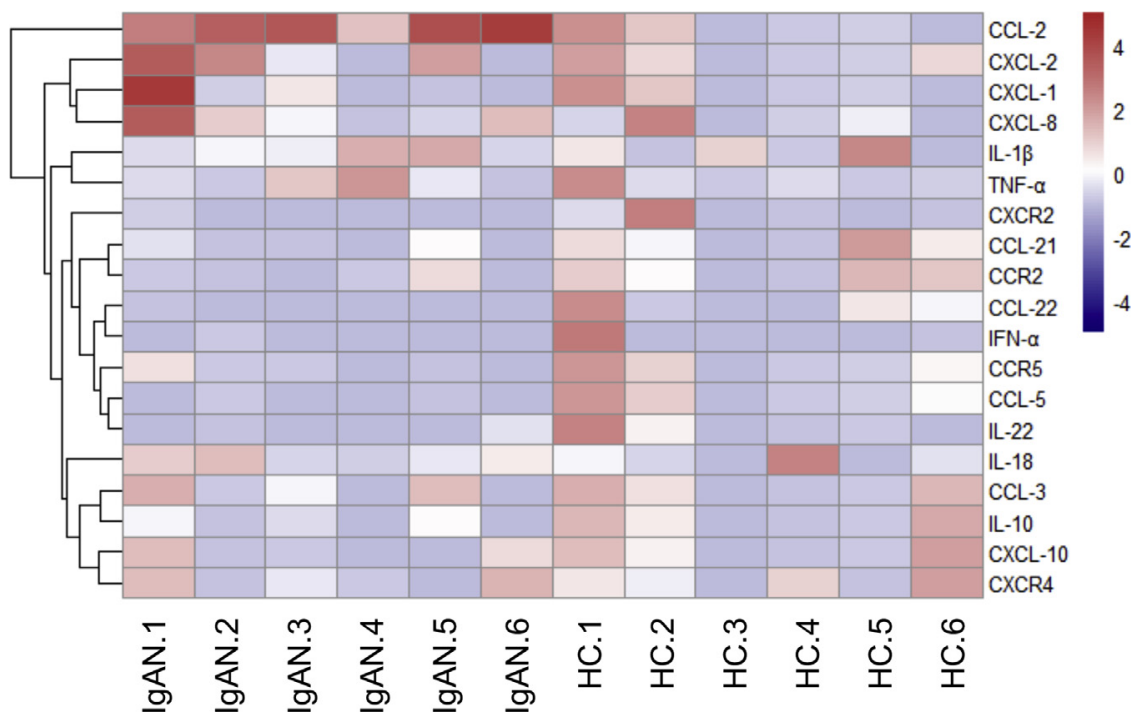

Figure 4 Expression profile of inflammatory response-related genes in exosomes from IgA nephropathy (IgAN) patients and healthy controls (HCs). Twenty cytokines and chemokines as well as receptors were screened in IgAN patients and matched controls. The expression patterns are presented as cluster heat maps established by the heat map package in $\mathrm{R}$ version 3.3.5. $n=6 \mathrm{IgAN}$ patients and $\mathrm{HCS}$. $\mathrm{CCL}$, chemokine ( $\mathrm{C}-\mathrm{C}$ motif) ligand; $\mathrm{CXCR}, \mathrm{C}-\mathrm{X}-\mathrm{C}$ chemokine receptor; IFN- $\alpha$, interferon- $\alpha$; TNF- $\alpha$, tumor necrosis factor- $\alpha$. healthy controls, as evidenced by Western blot analysis of exosome markers and renal cell markers (Figure 1C). Besides, exosome quantification was performed in $29 \operatorname{IgAN}$ patients and 15 healthy controls via EXOCET assay. The result confirmed remarkable increased exosome production in IgAN patients compared with controls (Figure 1D). The mass of exosomes was significantly higher in the urine from patients with severe proteinuria ( $>3.5 \mathrm{~g} / 24$ hours) compared with those without severe proteinuria $(<3.5 \mathrm{~g} / 24$ hours) in IgAN (Figure 1E).

\section{Urinary Exosome Excretion Is Correlated with Levels of} Proteinuria and Tubular Injury in IgAN Patients

Further analysis showed that the amount of exosomes correlated closely with levels of proteinuria $(r=0.935$, $P<0.05$ ) (Figure 2A). However, no correlation was found between urinary exosome excretion and eGFR in IgAN patients (Figure 2B). Because tubular epithelial cells are directly exposed to filtered protein in conditions of proteinuria and are the major cellular targets of proteinuria toxicity, ${ }^{3}$ the relationship between urinary exosome excretion and tubular injury was next examined. Urinary protein levels of neutrophil gelatinaseassociated lipocalin, a marker of tubular damage, were significantly correlated with proteinuria, as expected $(r=0.678, P<0.0001)$ (Figure $2 \mathrm{C}$ ); and a strong positive association was found between urinary exosome excretion and neutrophil gelatinase-associated lipocalin in $\operatorname{IgAN}$ patients $(r=0.594, P<0.05)$ (Figure 2D). These data suggest that urinary exosome production may attribute to proteinuria and the consequent tubular injury accruing from protein-overload toxicity.

\section{Correlation between Urinary Exosome Production and Kidney Histologic Injury in IgAN}

To further understand the relevance of exosomes to kidney histologic injury in IgAN, renal biopsy specimens were scored according to the Oxford Classification of IgA nephropathy $2016 .^{22}$ Representative kidney histologic injury,
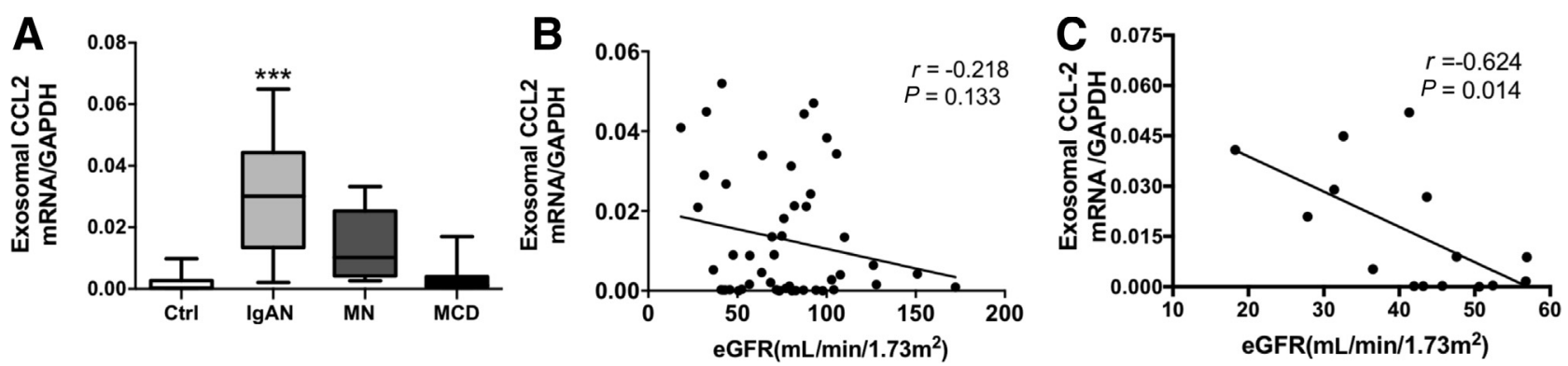

Figure 5 Exosomal chemokine (C-C motif) ligand 2 (CCL2) mRNA is up-regulated in IgA nephropathy (IgAN) patients and correlates with estimated glomerular filtration rate (eGFR). A: The relative expression of CCL2 mRNA in urinary exosomes of IgAN patients is significantly higher than in healthy controls (Ctrls) as well as minimal change disease (MCD) and membranous nephropathy (MN) patients. B and C: Lines show the linear correlation between CCL2 mRNA in urinary exosomes and eGFR in all IgAN patients and in patients with eGFR $<60 \mathrm{~mL} /$ minute per $1.73 \mathrm{~m}^{2} . n=55 \mathrm{IgAN}$ patients $(\mathbf{A}) ; n=24$ healthy controls (A); $n=9$ MCD patients (A); $n=16$ MN patients (A). ${ }^{* * *} P<0.001$ versus healthy controls. GAPDH, glyceraldehyde-3-phosphate dehydrogenase. 
A

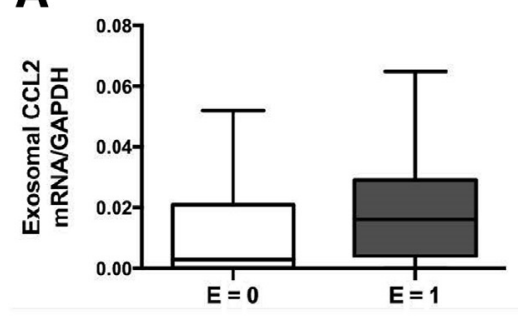

C

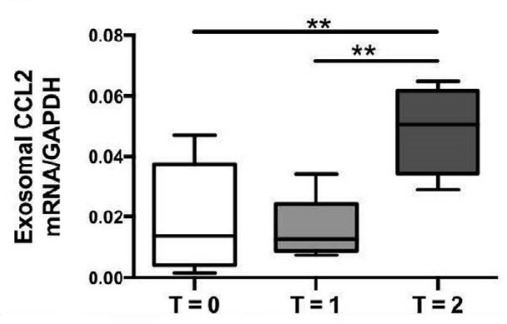

E

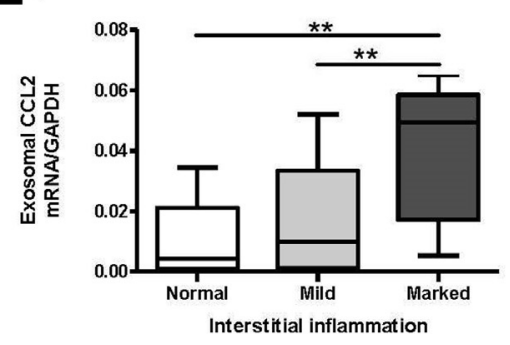

B

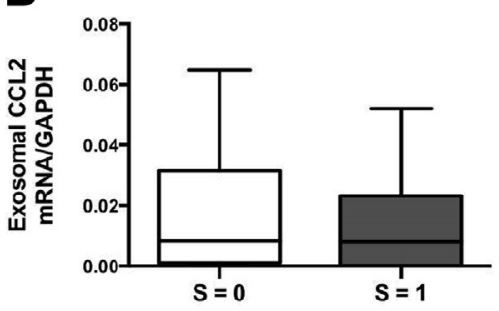

D

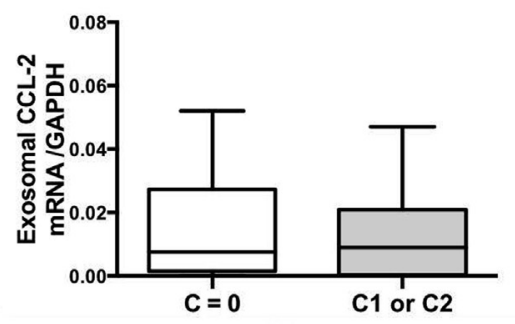

$\mathbf{F}$

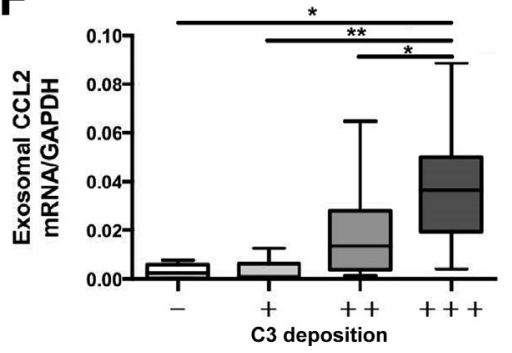

Figure 6 Exosomal chemokine ( $\mathrm{C}-\mathrm{C}$ motif) ligand 2 (CCL2) mRNA correlates with tubulointerstitial inflammation and fibrosis in the kidney. A and B: No differences in urinary exosomal CCL2 mRNA are found between IgA nephropathy $(\operatorname{IgAN})$ patients with $(E=1$ or $S=1)$ or without $(E=0$ or $S=0$ ) endocapillary hypercellularity (A) or segmental glomerulosclerosis (B). C: Patients with $>50 \%$ area of tubular atrophy and interstitial fibrosis $(T=2)$ show the highest expression of exosomal CCL2 mRNA ( $T=0$ indicates $<25 \%$; and $\mathrm{T}=1,25 \%$ to $50 \%)$. D: No significant difference is found between patients with $(C=1 / 2)$ or without $(C=0)$ crescents (cellular, fibrocellular, and fibrosis). E: The levels of CCL2 mRNA in urinary exosomes of patients with normal $(0 \%)$, mild $(<25 \%)$, or marked $(>25 \%)$ interstitial inflammation. Patients with marked interstitial inflammation show higher levels of urinary exosomal CCL2 mRNA. F: Urinary exosomal CCL2 mRNA expression of IgAN patients with different degrees of $\mathrm{C} 3$ deposition in mesangial areas. The cohort with +++ C3 complement deposition shows the highest expression of urinary exosomal CCL2 mRNA among all IgAN patients. $n=12$ patients with normal interstitial inflammation $(\mathbf{E}) ; n=25$ patients with mild interstitial inflammation (E); $n=8$ patients with marked interstitial inflammation $(\mathrm{E}) .{ }^{*} P<0.05$, ${ }^{* *} P<0.01$. GAPDH, glyceraldehyde-3-phosphate dehydrogenase. assessed by periodic acid-Schiff and Masson staining, is shown in Supplemental Figure S1. Among the enrolled patients, all presented with M1 lesions $(>50 \%$ of glomeruli hypercellular) and more urinary exosome excretion was observed compared with controls (Figure 1D); thus, no further subgroup was analyzed. When the cohort was stratified on the basis of endocapillary score, patients with endocapillary hypercellularity (E1) presented with increased urinary exosome production compared with patients without endocapillary hypercellularity (E0) $(P<0.001)$ (Figure 3A). However, no difference was found in patients with different degrees of segmental glomerulosclerosis and tubular atrophy/interstitial fibrosis (Figure 3, B and C). Patients with crescents $(\mathrm{C} 1 / \mathrm{C} 2)$ showed increased exosome excretion compared with those without crescents (C0) (Figure 3D). Thus, exosome release associates with more severe and active histologic activity (mesangial hypercellularity, endocapillary hypercellularity, and crescent).

\section{Exosomal Expression of Inflammation-Related Genes in IgAN Patients}

The expression profiles of inflammation-related genes were first analyzed in urinary exosomes from a screening set of IgAN patients and controls by real-time PCR $(n=6$ for each group). The clinical characteristics of patients and controls are listed in Table 2. Twenty key inflammatory cytokines and chemokines as well as their receptors were included in the analysis (Table 3). Among them, IL6 was not detectable in all samples, whereas most other genes showed low expression. By contrast, CCL2 was robustly upregulated in all IgAN samples, with a 10-fold induction compared with controls (Figure 4).

\section{Validation of Exosomal CCL2 mRNA in IgAN Patients}

To validate findings from the screening set, expression of CCL2 mRNA was further analyzed in exosome samples from 55 IgAN patients and 24 healthy controls. To determine the specificity of exosomal CCL2 in IgAN patients, 16 $\mathrm{MN}$ and $9 \mathrm{MCD}$ patients were also included as other glomerulopathy controls in this part. The basic clinical characteristics are shown in Supplemental Table S1. The groups of MCD and MN patients did not differ in age, sex, renal function, and levels of proteinuria compared with $\operatorname{IgAN}$ patients, although they exhibited significant proteinuria compared with healthy controls. Results confirmed that exosomal CCL2 is extremely highly overexpressed in IgAN patients compared with normal controls, as well as in MCD and $\mathrm{MN}$ patients (Figure 5A). Besides, a notable inverse correlation between exosomal CCL2 mRNA and eGFR was 
observed, especially in patients with eGFR $<60 \mathrm{~mL} /$ minute per $1.73 \mathrm{~m}^{2}(r=-0.624, P<0.05)$ (Figure 5, B and C).

\section{Exosomal CCL2 mRNA Correlates with Tubulointerstitial Inflammation}

To further explore the correlation between exosomal CCL2 mRNA with renal pathology, the levels of exosomal CCL2 mRNA were investigated in groups of patients stratified by MEST-C score. Levels of CCL2 mRNA expression did not vary between patients with and without endocapillary hypercellularity (Figure 6A) or segmental glomerulosclerosis (Figure 6B). However, CCL2 showed higher levels of expression in patients with more severe tubular atrophy and interstitial fibrosis $(P<0.01$ versus T0, $P<0.01$ versus T1) (Figure 6C). No significant differences in $C C L 2$ were found between patients with or without crescents (Figure 6D).

Tubulointerstitial inflammation was scored and stratified into three groups: normal, mild, and marked. ${ }^{16,18}$ CCL2 mRNA levels were progressively higher with increasing severity of tubulointerstitial inflammation $(P<0.01$ versus normal, $P<0.01$ versus mild) (Figure $6 \mathrm{E}$ ). $C C L 2$ mRNA levels also increased with increasing glomerular $\mathrm{C} 3$ deposition (Figure $6 \mathrm{~F})(P<0.05$ versus $\mathrm{C} 3-, P<0.01$ versus $\mathrm{C} 3+$, and $P<0.05$ versus $\mathrm{C} 3++$ ).

Thus, exosomal CCL2 mRNA is closely correlated with tubulointerstitial inflammation.

\section{Urinary Exosomes and Exosomal CCL2 mRNA as Biomarkers of IgAN}

To assess the diagnostic accuracy of exosomes and exosomal mRNA in urine, receiver operating characteristic curves were generated. Both urinary exosome quantification and CCL2 mRNA expression could efficiently discriminate IgAN from healthy controls, with areas under the receiver operating characteristic curve of 0.925 (95\% CI, 0.847-1) and 0.81 (95\% CI, 0.676-0.945), respectively. However, compared with urine exosome, exosomal CCL2 mRNA showed superiority in discriminating between IgAN patients with marked versus non-to-mild interstitial inflammation. The area under the receiver operating characteristic curve was 0.828 (95\% CI, 0.642-1.0) for exosomal CCL2 and 0.691 (95\% CI, 0.478-0.905) for exosomal protein (Figure 7).

\section{Urinary Exosome and Exosomal CCL2 mRNA as Predictors of IgAN Progression}

In total, 36 patients were monitored longitudinally, with an average follow-up period of 18.5 months after renal biopsy. The clinical characteristics of the patients at baseline and follow-up are shown in Table 4. Levels of proteinuria were significantly reduced during follow-up. Urinary exosomes from 14 patients among the follow-up group were analyzed.
Urinary exosome excretion decreased with the reduction in proteinuria (Figure 8, A and B). At the end of the follow-up, renal function, as measured by eGFR, declined in 25 patients $(69.4 \%)$. The remaining patients maintained stable renal function. Baseline urinary exosomal CCL2 expression was higher in patients whose IgAN progressed compared with the stable group, suggesting that CCL2 may be a reliable biomarker for predicting IgAN progression (Figure 8C).

\section{Discussion}

The clinical presentations of patients with IgAN are highly variable, ranging from asymptomatic microscopic hematuria to rapidly progressive glomerulonephritis. Noninvasive
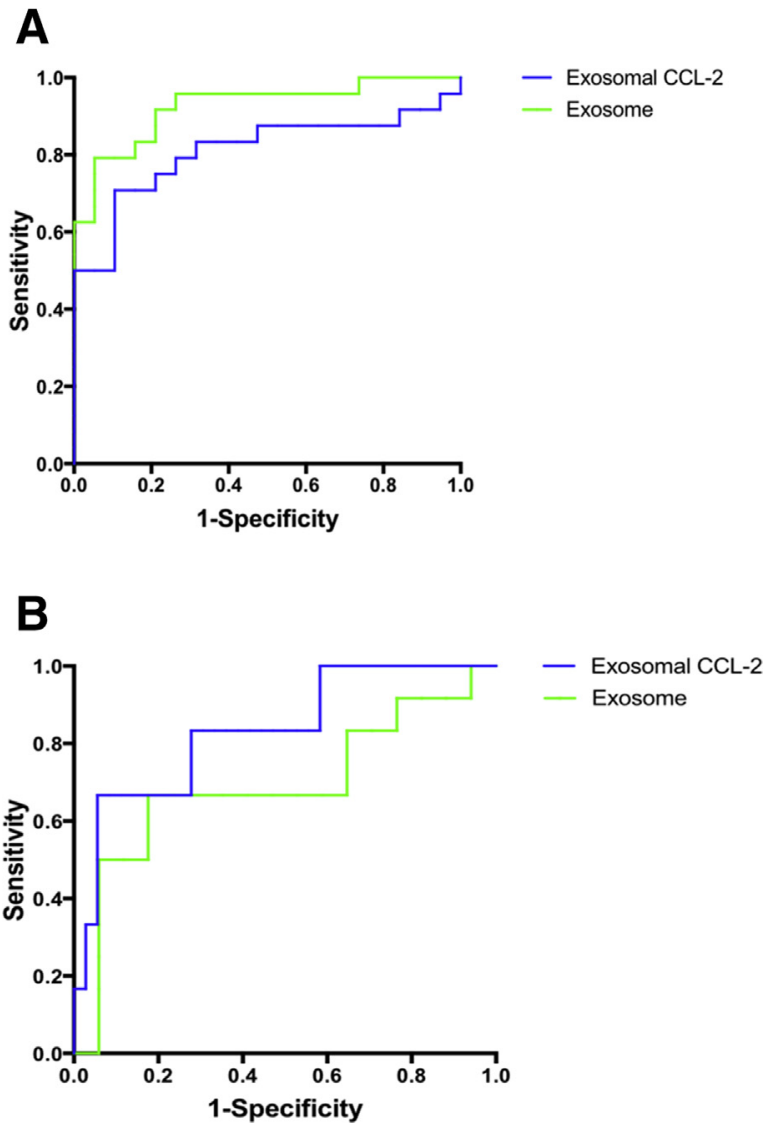

Figure 7 Urinary exosomes and exosomal chemokine (C-C motif) ligand 2 (CCL2) mRNA as biomarkers of IgA nephropathy (IgAN). A: Discrimination of IgAN from controls. Receiver operating characteristic (ROC) curves were generated using urinary exosome quantification and CCL2 mRNA. The areas under the ROC curve (AUCs) were 0.925 (95\% CI, 0.847-1) and 0.81 (95\% CI, 0.676-0.945) for exosome number and CCL2, respectively. B: Discrimination of interstitial inflammation. ROC curves of urinary exosome quantification and CCL2 mRNA were generated for distinguishing the severity of interstitial inflammation among IgAN patients. The AUCs were $0.828(95 \%$ CI, $0.642-1.0)$ and 0.691 (95\% CI, 0.478-0.905) for exosomal CCL2 and exosome quantification, respectively. $n=29 \mathrm{IgAN}$ patients using urinary exosome quantification $(\mathbf{A}) ; n=15$ healthy controls using urinary exosome quantification (A); $n=55$ IgAN patients using CCL2 mRNA (A); $n=24$ healthy controls using $C C L 2$ mRNA (A). 
Table 4 Human Subject Characteristics of IgAN Patients with Follow-Up

\begin{tabular}{lll}
\hline Characteristic & Baseline & Follow-up \\
\hline Male:female, $n$ & $18: 18$ & $18: 18$ \\
Age, years & $40.1 \pm 13.5$ & $40.1 \pm 13.5$ \\
$\mathrm{BUN}, \mathrm{mmol} / \mathrm{L}$ & $5.6(4.3-7.0)$ & $6.2(4.6-9.4)$ \\
$\mathrm{SCr}, \mu \mathrm{mol} / \mathrm{L}$ & $91.5(75.5-136.2)$ & $104(82-198)$ \\
$\mathrm{UA}, \mu \mathrm{mol} / \mathrm{L}$ & $341(271-433)$ & $363(242-590)$ \\
eGFR & $78.5(42.3-93.5)$ & $70.5(31.4-87.3)$ \\
Proteinuria, & $2.67(1.32-6.45)$ & $0.69(0.25-1.26)^{*}$ \\
$\quad$ g/24 hours & & \\
Follow-up period, & $18.5(10-33)$ & \\
$\quad$ & & \\
\hline
\end{tabular}

Data are expressed as means $\pm S D$ or median (interquartile range). ${ }^{*} P<0.001$ (U-test).

BUN, blood urea nitrogen; eGFR, estimated glomerular filtration rate; IgAN, IgA nephropathy; SCr, serum creatinine; UA, uric acid.

biomarkers reflecting histologic changes are, therefore, crucial for predicting disease progression and determining therapeutic strategy. Although persistent proteinuria can reflect active capillary and podocyte lesions, proteinuria by itself cannot discriminate between active inflammation and chronic damage. ${ }^{18,23}$ In this study, we explored the role of urinary exosome protein and exosomal inflammatory response-related mRNA in the diagnosis of IgAN and their correlation with renal histologic damage.

First, it was found that urinary exosome excretion was elevated in IgAN patients compared with healthy controls. Moreover, exosome excretion was strongly correlated with levels of proteinuria and the severity of tubular injury. In the follow-up study, a decline in exosome excretion paralleled the attenuation of proteinuria. Regarding specific renal pathologies, exosome excretion was increased in patients with endocapillary hypercellularity and higher severity of crescents. Thus, it is possible that in proteinuric conditions, higher levels of proteinuria with tubular injury and active proliferative lesions (higher mesangial score, crescent, and endocapillary hypercellularity) contribute to exosome release. The increased exosome production might be originating from the proliferative cells. Then, the release of exosomes seems to decrease as injury progresses toward end stage with renal fibrosis. Thus, urinary exosome may be a sensitive fluid-biopsy indicator of disease activity in IgAN patients.

Previously, increased exosome release has been reported in hypoxia, acidic $\mathrm{pH}$, heat shock, and oxidative stress conditions. $^{24,25}$ Megalin/cubulin-mediated albumin reabsorption and subsequent lysosome rupture are observed in tubular epithelial cells exposed to albumin. ${ }^{26}$ Tubular epithelial cells under bovine serum albumin exposure show increased exosome release, consistent with our findings in clinical samples. ${ }^{20}$ During exosome biogenesis, multivesicular bodies fuse with the plasma membrane and exosome is released. Alternatively, multivesicular bodies can fuse with lysosomes for degradation. ${ }^{27}$ In conditions of proteinuria, it was suspected that proteinuria may increase exosome release because of the dysfunction of lysosomes in tubular epithelial cells. However, the mechanisms by which exosome excretion is enhanced in conditions of proteinuria require further investigation. As an alternative, it is possible that the proliferation of renal epithelial cells in patients with greater histologic activity may contribute to exaggerated exosome formation and release.

Second, among 20 selected inflammatory responserelated genes, mRNA for CCL2 was markedly induced in urinary exosomes from IgAN patients compared with controls. More important, levels of exosomal CCL2 mRNA correlated with the severity of tubular atrophy and interstitial fibrosis, and interstitial inflammation, especially macrophage infiltration documented by renal biopsy. A significant inverse correlation between CCL2 and eGFR was found in IgAN patients. Because tubulointerstitial damage is a key histopathological risk factor for deterioration in renal function, urinary exosomal CCL2 may be a useful predictive biomarker for progression of IgAN. Accordingly, in our
A

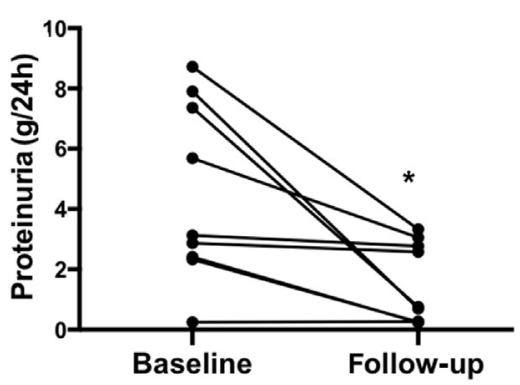

B

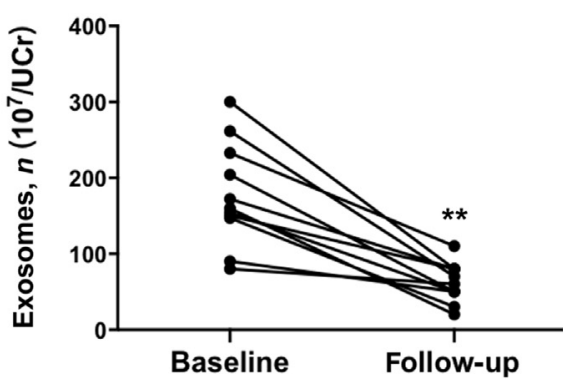

C

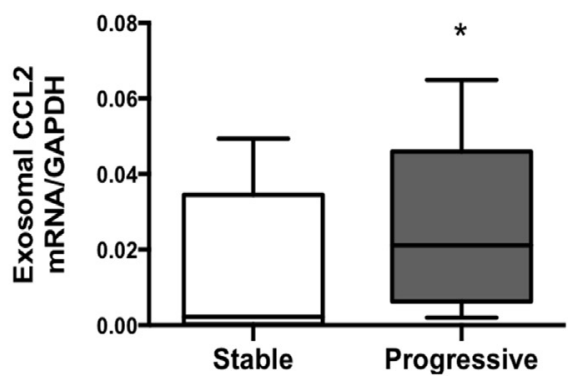

Figure 8 Urinary exosomal protein and chemokine (C-C motif) ligand 2 (CCL2) mRNA during follow-up of IgA nephropathy patients. Urine samples were collected from patients at the time of renal biopsy (baseline) and on follow-up at an average of 18.5 months. A: Proteinuria significantly decreases after treatment compared with baseline. B: Urinary exosomes were obtained from a subset of patients during the follow-up period. In these patients, urinary exosomal excretion reduces compared with baseline. C: Baseline urinary exosomal CCL2 expression is higher in progressive patients [estimated glomerular filtration rate (eGFR) decline] compared with the stable group (no decline of eGFR). $n=36$ (A); $n=14$ (B); $n=25$ eGFR decline group (C); $n=11$ no decline of eGFR group $(\mathbf{C}) .{ }^{*} P<0.05,{ }^{*} P<0.01$ ( $t$-test or $U$-test for non-non-parametric data). GAPDH, glyceraldehyde-3-phosphate dehydrogenase; UCr, urine creatinine. 
follow-up cohort, higher baseline exosomal CCL2 levels associated with progressive renal function deterioration. Thus, exosomal CCL2 correlates specifically with renal interstitial injury and may have value as a predictor of disease progression in IgAN.

Monocyte chemotactic peptide-1 (CCL2) is a well-studied chemokine that promotes macrophage infiltration and the development of renal disease. Eardley et $\mathrm{al}^{28}$ found that the recruitment of macrophages by CCL2 links albuminuria to the development of renal injury in the setting of IgAN. Accordingly, urinary CCL2 has been shown to be a prognostic marker of end-stage renal disease for patients with IgAN. ${ }^{29}$ Urinary $C C L 2$ from primary patients with glomerulonephritis has correlated with tubular atrophy and interstitial fibrosis in these diseases. ${ }^{30}$ In this study, we determined that urinary exosomal CCL2 mRNA is a novel biomarker of tubulointerstial injury in IgAN. More important, it might be a specific marker to discriminate IgAN from MCD and MN, although validation studies are needed in a large cohort of patients. However, the underlying mechanism through which exosomal $C C L 2$ participates in disease pathogenesis will require further investigation. Nevertheless, it was found that exosomal CCL2 mRNA correlated with levels of $\mathrm{C} 3$ deposition, and complement activation may orchestrate chronic inflammation in IgAN. Immune complex deposition triggers complement activation, driving mesangial cells to proliferate and produce cytokines and chemokines. Thus, $\mathrm{C} 3$ deposition might underpin the initial inflammatory response, including the generation of exosomal $C C L 2$ that can further amplify kidney inflammation.

\section{Conclusion}

Herein, we have demonstrated that patients with IgAN have augmented urinary excretion of exosomes corresponding to higher renal pathology severity and proteinuria. CCL2 mRNA pacing in urinary exosomes correlates closely with tubulointerstitial injury and progressive deterioration in renal function. Thus, urinary exosomes and CCL2 mRNA may serve as novel and promising biomarkers to predict IgAN progression.

\section{Supplemental Data}

Supplemental material for this article can be found at https://doi.org/10.1016/j.ajpath.2018.07.017.

\section{References}

1. Berthelot L, Robert T, Vuiblet V, Tabary T, Braconnier A, Drame M, Toupance O, Rieu P, Monteiro RC, Toure F: Recurrent IgA nephropathy is predicted by altered glycosylated $\operatorname{IgA}$, autoantibodies and soluble CD89 complexes. Kidney Int 2015, 88:815-822

2. Zhao YF, Zhu L, Liu LJ, Shi SF, Lv JC, Zhang H: Measures of urinary protein and albumin in the prediction of progression of $\operatorname{IgA}$ nephropathy. Clin J Am Soc Nephrol 2016, 11:947-955
3. Roberts IS: Pathology of IgA nephropathy. Nat Rev Nephrol 2014, 10 : 445-454

4. Serino G, Pesce F, Sallustio F, De Palma G, Cox SN, Curci C, Zaza G, Lai KN, Leung JC, Tang SC, Papagianni A, Stangou M, Goumenos D, Gerolymos M, Takahashi K, Yuzawa Y, Maruyama S, Imai E, Schena FP: In a retrospective international study, circulating miR-148b and let-7b were found to be serum markers for detecting primary IgA nephropathy. Kidney Int 2016, 89:683-692

5. Thery C, Zitvogel L, Amigorena S: Exosomes: composition, biogenesis and function. Nat Rev Immunol 2002, 2:569-579

6. Tkach M, Thery C: Communication by extracellular vesicles: where we are and where we need to go. Cell 2016, 164:1226-1232

7. Wei PZ, Kwan BC, Chow KM, Cheng PM, Luk CC, Li PK, Szeto CC: Urinary mitochondrial DNA level is an indicator of intra-renal mitochondrial depletion and renal scarring in diabetic nephropathy. Nephrol Dial Transplant 2018, 33:784-788

8. Pisitkun T, Shen RF, Knepper MA: Identification and proteomic profiling of exosomes in human urine. Proc Natl Acad Sci U S A 2004, 101:13368-13373

9. Khurana R, Ranches G, Schafferer S, Lukasser M, Rudnicki M, Mayer G, Huttenhofer A: Identification of urinary exosomal noncoding RNAs as novel biomarkers in chronic kidney disease. RNA 2017, 23: $142-152$

10. Lv LL, Cao YH, Pan MM, Liu H, Tang RN, Ma KL, Chen PS, Liu BC: $\mathrm{CD} 2 \mathrm{AP}$ mRNA in urinary exosome as biomarker of kidney disease. Clin Chim Acta 2014, 428:26-31

11. Lv LL, Cao YH, Ni HF, Xu M, Liu D, Liu H, Chen PS, Liu BC: MicroRNA-29c in urinary exosome/microvesicle as a biomarker of renal fibrosis. Am J Physiol Ren Physiol 2013, 305:F1220-F1227

12. Eissa S, Matboli M, Aboushahba R, Bekhet MM, Soliman Y: Urinary exosomal microRNA panel unravels novel biomarkers for diagnosis of type 2 diabetic kidney disease. J Diabetes Complications 2016, 30: $1585-1592$

13. Mohan A, Singh RS, Kumari M, Garg D, Upadhyay A, Ecelbarger CM, Tripathy S, Tiwari S: Urinary exosomal microRNA$451-5 \mathrm{p}$ is a potential early biomarker of diabetic nephropathy in rats. PLoS One 2016, 11:e0154055

14. Sole C, Cortes-Hernandez J, Felip ML, Vidal M, Ordi-Ros J: miR-29c in urinary exosomes as predictor of early renal fibrosis in lupus nephritis. Nephrol Dial Transplant 2015, 30:1488-1496

15. Yeo SC, Cheung CK, Barratt J: New insights into the pathogenesis of IgA nephropathy. Pediatr Nephrol 2018, 33:763-777

16. Myllymaki JM, Honkanen TT, Syrjanen JT, Helin HJ, Rantala IS, Pasternack AI, Mustonen JT: Severity of tubulointerstitial inflammation and prognosis in immunoglobulin A nephropathy. Kidney Int 2007, 71:343-348

17. Freese P, Norden G, Nyberg G: Morphologic high-risk factors in IgA nephropathy. Nephron 1998, 79:420-425

18. Myllymaki J, Honkanen T, Syrjanen J, Helin H, Rantala I, Pasternack A, Mustonen J: Uric acid correlates with the severity of histopathological parameters in IgA nephropathy. Nephrol Dial Transplant 2005, 20:89-95

19. Yokoyama H, Wada T, Furuichi K, Segawa C, Shimizu M, Kobayashi K, Su S, Mukaida N, Matsushima K: Urinary levels of chemokines (MCAF/MCP-1, IL-8) reflect distinct disease activities and phases of human IgA nephropathy. J Leukoc Biol 1998, 63: 493-499

20. Lv LL, Feng Y, Wen Y, Wu WJ, Ni HF, Li ZL, Zhou LT, Wang B, Zhang JD, Crowley SD, Liu BC: Exosomal CCL2 from tubular epithelial cells is critical for albumin-induced tubulointerstitial inflammation. J Am Soc Nephrol 2018, 29:919-935

21. Thomou T, Mori MA, Dreyfuss JM, Konishi M, Sakaguchi M, Wolfrum C, Rao TN, Winnay JN, Garcia-Martin R, Grinspoon SK, Gorden P, Kahn CR: Adipose-derived circulating miRNAs regulate gene expression in other tissues. Nature 2017, 542:450-455

22. Trimarchi H, Barratt J, Cattran DC, Cook HT, Coppo R, Haas M, Liu ZH, Roberts IS, Yuzawa Y, Zhang H, Feehally J; IgAN 
Classification Working Group of the International IgA Nephropathy Network and the Renal Pathology Society; Conference Participants: Oxford classification of IgA nephropathy 2016: an update from the IgA nephropathy classification working group. Kidney Int 2017, 91: 1014-1021

23. Coppo R, Fervenza FC: Persistent microscopic hematuria as a risk factor for progression of $\operatorname{IgA}$ nephropathy: new floodlight on a nearly forgotten biomarker. J Am Soc Nephrol 2017, 28:2831-2834

24. Li L, Li C, Wang S, Wang Z, Jiang J, Wang W, Li X, Chen J, Liu K, Li C, Zhu G: Exosomes derived from hypoxic oral squamous cell carcinoma cells deliver miR-21 to normoxic cells to elicit a prometastatic phenotype. Cancer Res 2016, 76:1770-1780

25. de Jong OG, Verhaar MC, Chen Y, Vader P, Gremmels H, Posthuma G, Schiffelers RM, Gucek M, van Balkom BW: Cellular stress conditions are reflected in the protein and RNA content of endothelial cell-derived exosomes. J Extracell Vesicles 2012, 1. 18396

26. Liu D, Wen Y, Tang TT, Lv LL, Tang RN, Liu H, Ma KL, Crowley SD, Liu BC: Megalin/cubulin-lysosome-mediated albumin reabsorption is involved in the tubular cell activation of NLRP3 inflammasome and tubulointerstitial inflammation. J Biol Chem 2015, 290:18018-18028

27. Hurley JH, Odorizzi G: Get on the exosome bus with ALIX. Nat Cell Biol 2012, 14:654-655

28. Eardley KS, Zehnder D, Quinkler M, Lepenies J, Bates RL, Savage CO, Howie AJ, Adu D, Cockwell P: The relationship between albuminuria, MCP-1/CCL2, and interstitial macrophages in chronic kidney disease. Kidney Int 2006, 69: 1189-1197

29. Torres DD, Rossini M, Manno C, Mattace-Raso F, D’Altri C, Ranieri E, Pontrelli P, Grandaliano G, Gesualdo L, Schena FP: The ratio of epidermal growth factor to monocyte chemotactic peptide-1 in the urine predicts renal prognosis in IgA nephropathy. Kidney Int 2008, 73:327-333

30. Worawichawong S, Worawichawong S, Radinahamed P, Muntham D, Sathirapongsasuti N, Nongnuch A, Assanatham M, Kitiyakara C: Urine epidermal growth factor, monocyte chemoattractant protein-1 or their ratio as biomarkers for interstitial fibrosis and tubular atrophy in primary glomerulonephritis. Kidney Blood Press Res 2016, 41: 997-1007 